\title{
On Newton-Cartan local renormalization group and anomalies
}

\author{
Roberto Auzzi, ${ }^{a, b}$ Stefano Baiguera, ${ }^{a}$ Francesco Filippini ${ }^{a}$ and Giuseppe Nardelli ${ }^{a, c}$ \\ ${ }^{a}$ Dipartimento di Matematica e Fisica, Università Cattolica del Sacro Cuore, \\ Via Musei 41, 25121 Brescia, Italy \\ ${ }^{b}$ INFN Sezione di Perugia, \\ Via A. Pascoli, 06123 Perugia, Italy \\ ${ }^{c}$ TIFPA - INFN, c/o Dipartimento di Fisica, Università di Trento, \\ 38123 Povo (TN), Italy \\ E-mail: roberto.auzzi@unicatt.it, stefano.baiguera92@gmail.com, \\ francesco.filippini@hotmail.it, giuseppe.nardelli@unicatt.it
}

ABSTRACT: Weyl consistency conditions are a powerful tool to study the irreversibility properties of the renormalization group. We apply this formalism to non-relativistic theories in 2 spatial dimensions with boost invariance and dynamical exponent $z=2$. Different possibilities are explored, depending on the structure of the gravitational background used as a source for the energy-momentum tensor.

KEYwords: Anomalies in Field and String Theories, Renormalization Group, Space-Time Symmetries

ArXiv ePrint: 1610.00123 


\section{Contents}

1 Introduction 1

2 Preliminaries 3

2.1 Newton-Cartan geometry 3

2.2 Sources 5

2.3 The trace anomaly without Frobenius condition 6

$\begin{array}{ll}2.4 & \text { The trace anomaly with Frobenius condition }\end{array}$

3 Local RG without Frobenius condition and $N_{n}=0 \quad 8$

4 Local RG with Frobenius condition $\quad 10$

$5 \quad$ The $N_{n}>0$ sectors $\quad 12$

5.1 The sector $N_{n}=1 \quad 13$

5.2 Cohomological problem of the $N_{n}=1$ sector 13

$\begin{array}{lll}5.3 & \text { Sectors with higher } N_{n} & 15\end{array}$

6 Conclusions 16

$\begin{array}{ll}\text { A Local counterterms in the Frobenius case } & 17\end{array}$

$\begin{array}{ll}\text { B Consistency conditions with Frobenius conditions } & 19\end{array}$

\section{Introduction}

Relativistic trace anomalies (see [1] for a review) give non-trivial constraints on the possible infrared (IR) dynamics which can emerge from an ultraviolet (UV) unitary theory. In 2 dimensions this is established by Zamolodchikov $c$-theorem [2]. In 4 dimensions the monotonicity property of the anomaly coefficient $a$ ( $a$-theorem) was first conjectured in [3]; a perturbative proof was given by [4-6] with the local renormalization group (RG) equations. A proof using dispersion relations was given in $[7,8]$.

The local RG equations [4-6] are derived imposing the Wess-Zumino (WZ) consistency conditions for the trace anomaly $[9,10]$ of the theory in a generic gravity background with spacetime-dependent couplings. It is a very useful tool to study relativistic RG flows nearby conformal fixed points in various dimensions [11-16] and it has also interesting applications in the supersymmetric case $[13,17,18]$. For reviews see $[19,20]$.

Genuine anomalies correspond to terms which are intrinsic properties of the field theory and do not depend on the choice of counterterms used in the renormalization procedure. There are other violations of conformal symmetries in curved space which instead are 
counterterm-dependent. For example, in the relativistic case in 4 dimensions the trace of the energy-momentum tensor contains the following terms:

$$
T_{\mu}^{\mu} \supset a E_{4}-c W^{2}+b R^{2}-\frac{2}{3} a^{\prime} \square R .
$$

The anomaly coefficient $a$ coincides, at the fixed point, with the decreasing function in the $a$ theorem, while $c$ has no monotonicity property [21]. The coefficient $b$ vanishes at conformal fixed points due to the Wess-Zumino consistency conditions. Moreover, as we will show, it is possible to choose counterterms in such a way that it vanishes along a specific RG flow trajectory which interpolates between an UV and an IR fixed point. The $a^{\prime}$ coefficient can be shifted by a counterterm in the vacuum functional which is proportional to $R^{2}$ and so it is not a genuine scheme-indepedendent anomaly. However, this term could still play a role in the monotonocity properties of the RG flow. For example, following [22, 23] one may consider the difference between the UV and IR $a^{\prime}$ coefficient $\Delta a^{\prime}=a_{U V}^{\prime}-a_{I R}^{\prime}$ along a given RG flow trajectory; in a scheme where $b=0$ along the RG flow, this is a monotonicallydecreasing quantity. Note that it explicitly depends on the given RG trajectory and not just on its end points. In $[22,23]$ it was conjectured that the minimum of $\Delta a^{\prime}$ among all the possible RG flows coincides with $\Delta a=a_{U V}-a_{I R}$.

It is interesting to explore trace anomalies also in the non-relativistic case, in order to study non-trivial constraints on the renormalization group flow. ${ }^{1}$ At a generic scaleinvariant fixed point, various relative scaling of space and time, which are parameterized by the dynamical exponents $z$, are in principle allowed i.e.

$$
x^{i} \rightarrow e^{\sigma} x^{i}, \quad t \rightarrow e^{z \sigma} t .
$$

Moreover, the details of the anomaly structure depend crucially on the symmetry content of the theories, in particular if we require or not boost invariance and also if we require or not integrability of time slices of the gravitational background (Frobenius condition). This condition is important for causality when the gravitational background is physical; on the other hand, in order to define the energy-momentum tensor, we need to consider generic variations of the background metric, including the ones which do not satisfy the Frobenius condition.

Scale anomalies in theories without boost invariance (Lifshitz) were studied by several authors, e.g. [24-29]. It turns out that, in all the cases that have been studied so far, the scheme-independent trace anomalies at the fixed point have vanishing Weyl variation (type $B[30]^{2}$ ).

In this paper we will be interested in the case with boost invariance. The natural background is provided by the Newton-Cartan (NC) gravity. Two different attitudes concerning trace anomalies are possible:

\footnotetext{
${ }^{1}$ One may wonder if a non-relativistic trace anomaly can be obtained as an infrared limit of a relativistic one. This is not the case because the relativistic scale symmetry is explicitly broken by the mass gap which is necessary to have a non-relativistic limit. Indeed relativistic trace anomaly coefficients count the number of massless degrees of freedom, which is zero for a $z \neq 1$ fixed point. The non-relativistic scale symmetry is an IR emergent phenomenon which does not exist in the far relativistic UV.

${ }^{2}$ Anomalies with non-trivial Weyl variation are instead called of type $A$; they correspond to non-trivial solutions of the Wess-Zumino consistency conditions.
} 
- The case where causality is not required on the gravity background; this setting was first studied by [31]. In this case the anomaly in $2+1$ dimensions and for $z=2$ has a very rich structure, because an infinite number of terms can be written by dimensional analysis. All these terms live in separated sectors, which are labelled by the integer $N_{n}$. A Weyl variation does not change the value of $N_{n}$ and the WZ consistency conditions can be studied independently in each separated sector, where just a finite number of terms is present. In particular, in the simplest sector $N_{n}=0$ the anomaly structure is identical to the trace anomaly of relativistic theories in 4 dimensions and a natural type $A$ candidate for a monotonicity theorem is the coefficient of the $E_{4}$ term. The calculation of the $N_{n}=0$ anomaly in the case of a free scalar was recently done in [32].

- The case in which the Frobenius condition is imposed has a much simpler anomaly structure. For $d=2+1$ and $z=2$, the number of terms allowed by dimensional analysis is finite [33] and the only scheme-independent anomaly turns out to be of type $B[28,33]$.

The purpose of the present paper is to initiate an analysis, using the local RG formalism, of the non-relativistic scale anomalies in theories with Galilean boost invariance. We focus on the $d=2+1$ and $z=2$ case, and we explore both the case with and without Frobenius condition:

- The structure of the local RG equation in the simplest sector $N_{n}=0$ of the case without Frobenius conditions turns out to be the same as for the relativistic theories in 4 dimensions.

- If we impose the Frobenius conditions, there is no scheme-independent $a$-theorem candidate. However it is still interesting to study the local RG equations; for example it might be possible to identify monotonic scheme-dependent quantities analog to the $a^{\prime}$ of the relativistic four-dimensional case. A similar study, in the case without boost invariance, was done in [29].

We also study in detail the $N_{n}=1$ sector of the anomaly without Frobenius conditions and we find that no anomaly is allowed by WZ consistency condition. We leave the study of higher sectors with $N_{n}>1$ as a challenging problem for further investigation; it could be that these sectors contain some interesting candidates for monotonicity theorems.

\section{Preliminaries}

\subsection{Newton-Cartan geometry}

The Newton-Cartan (NC) gravity is a covariant version of Newtonian gravity; putting non-relativistic theories in a Newton-Cartan gravitational background is a very useful tool in condensed-matter physics because it gives the natural sources for the operators in the energy-momentum tensor multiplet, e.g. [34-41]. 
A NC gravity background is defined by the tensors $h_{\alpha \beta}, h^{\mu \nu}$, and by the vectors $v^{\mu}$ and $n_{\mu}$ with properties:

$$
\begin{aligned}
n_{\mu} h^{\mu \alpha} & =0, & n_{\mu} v^{\mu} & =1, \\
h^{\mu \alpha} h_{\alpha \nu} & =\delta_{\nu}^{\mu}-v^{\mu} n_{\nu}=P_{\nu}^{\mu}, & h_{\mu \alpha} v^{\alpha} & =0 .
\end{aligned}
$$

As a further ingredient, a $\mathrm{U}(1)$ gauge potential $A_{\mu}$ for the number particle symmetry must be introduced. Causality is specified by the Frobenius condition, which takes the form

$$
d n \wedge n=0,
$$

where the 1-form $n=n_{\mu} d x^{\mu}$ specifies the time direction.

The symmetries of NC gravity include a local version of Galilean boosts, which are called Milne boosts and are given by the following shifts, parameterized by the functions $\psi_{\mu}\left(x^{\alpha}\right)$ :

$$
\begin{aligned}
v^{\prime \mu} & =v^{\mu}+h^{\mu \nu} \psi_{\nu} \\
h_{\mu \nu}^{\prime} & =h_{\mu \nu}-\left(n_{\mu} P_{\nu}^{\rho}+n_{\nu} P_{\mu}^{\rho}\right) \psi_{\rho}+n_{\mu} n_{\nu} h^{\rho \sigma} \psi_{\rho} \psi_{\sigma}, \\
A_{\mu}^{\prime} & =A_{\mu}+P_{\mu}^{\rho} \psi_{\rho}-\frac{1}{2} n_{\mu} h^{\alpha \beta} \psi_{\alpha} \psi_{\beta} .
\end{aligned}
$$

As a tool to write Milne boost invariant quantities in a systematic way, we use the null reduction from a relativistic parent space, ${ }^{3}$ as introduced in [42]:

$$
G_{M N}=\left(\begin{array}{cc}
0 & n_{\mu} \\
n_{\nu} & n_{\mu} A_{\nu}+n_{\nu} A_{\mu}+h_{\mu \nu}
\end{array}\right), \quad G^{M N}=\left(\begin{array}{cc}
A^{2}-2 v \cdot A & v^{\mu}-h^{\mu \sigma} A_{\sigma} \\
v^{\nu}-h^{\nu \sigma} A_{\sigma} & h^{\mu \nu}
\end{array}\right),
$$

where the null reduction is taken along the direction:

$$
n^{M}=(1,0, \ldots), \quad n_{M}=\left(0, n_{\mu}\right)
$$

We will refer to this trick as Discrete Light-Cone Quantization (DLCQ). Let $D_{A}, R$, $R_{A B C D}, R_{A B}$ denote respectively the covariant derivative, the scalar curvature and the Riemann and Ricci tensors defined by the Levi-Civita connection from the metric in eq. (2.5). The spacetime volume element is defined as:

$$
\sqrt{g}=\sqrt{\operatorname{det}\left(n_{\mu} n_{\nu}+h_{\mu \nu}\right)}=\sqrt{-\operatorname{det} G_{A B}} .
$$

Local Weyl transformations are parameterized by a function $\sigma$. In the DLCQ formalism $\sigma$ is taken independent from the null direction:

$$
n^{A} D_{A} \sigma=0 .
$$

In our conventions, the Weyl scaling of the extra-dimensional metric and of the $\mathrm{NC}$ objects is:

$$
G_{M N} \rightarrow e^{2 \sigma} G_{M N}, \quad n_{\mu} \rightarrow e^{2 \sigma} n_{\mu}, \quad h_{\mu \nu} \rightarrow e^{2 \sigma} h_{\mu \nu} .
$$

Here and in the rest of the paper we specialize to dynamical exponent $z=2$.

\footnotetext{
${ }^{3}$ Capital latin indices will always refer to $d+2$-dimensional tensors, while greek ones to $d+1$-dimensional objects.
} 


\subsection{Sources}

The allowed perturbations on the background fields must satisfy eqs. (2.1). The most general variation is parameterized by an arbitrary $\delta n_{\mu}$, a transverse perturbation $\delta u^{\mu}$ with $\delta u^{\mu} n_{\mu}=0$ and a transverse metric perturbation $\delta \tilde{h}^{\alpha \beta} n_{\beta}=0$. In term of these quantities, the variations of the background fields read:

$$
\delta A_{\mu}, \quad \delta n_{\mu}, \quad \delta v^{\mu}=-v^{\mu} v^{\alpha} \delta n_{\alpha}+\delta u^{\mu}, \quad \delta h^{\mu \nu}=-v^{\mu} \delta n^{\nu}-\delta n^{\mu} v^{\nu}-\delta \tilde{h}^{\mu \nu} .
$$

At the linear order nearby the flat limit, eq. (2.10) gives: ${ }^{4}$

$$
\begin{aligned}
& n_{\mu}=\left(1+\delta n_{0}, \delta n_{i}\right), \quad v^{\mu}=\left(1-\delta n_{0}, \delta u_{i}\right), \quad \delta \tilde{h}^{0 i}=0, \\
& h_{\mu \nu}=\left(\begin{array}{cc}
0 & -\delta u_{i} \\
-\delta u_{i} & \delta_{i j}+\delta \tilde{h}_{i j}
\end{array}\right), \quad h^{\mu \nu}=\left(\begin{array}{cc}
0 & -\delta n_{i} \\
-\delta n_{i} & \delta_{i j}-\delta \tilde{h}_{i j}
\end{array}\right) .
\end{aligned}
$$

In term of the null reduction fields, this corresponds to:

$$
\begin{aligned}
G_{A B} & =\left(\begin{array}{ccc}
0 & 1+\delta n_{0} & \delta n_{i} \\
1+\delta n_{0} & 2 \delta A_{0} & \delta A_{i}-\delta u_{i} \\
\delta n_{i} & \delta A_{i}-\delta u_{i} & \delta_{i j}+\delta \tilde{h}_{i j}
\end{array}\right) \\
G^{A B} & =\left(\begin{array}{ccc}
-2 \delta A_{0} & 1-\delta n_{0} & -\delta A_{i}+\delta u_{i} \\
1-\delta n_{0} & 0 & -\delta n_{i} \\
-\delta A_{i}+\delta u_{i} & -\delta n_{i} & \delta_{i j}+\delta \tilde{h}_{i j}
\end{array}\right) .
\end{aligned}
$$

These sources are useful to define conserved currents. We will consider the vacuum functional $W\left[g_{\mu \nu}\right]$ :

$$
e^{i W\left[G_{M N}\right]}=\int \mathcal{D} \phi e^{i S\left[\phi, G_{M N}\right]}
$$

where $\phi$ runs over the dynamical fields of the theory. The expectation values of the energymomentum tensor multiplet are defined by:

$$
\delta W=\int d^{d+1} x \sqrt{g}\left(\frac{1}{2} T_{i j} \delta \tilde{h}_{i j}+j^{\mu} \delta A_{\mu}-\epsilon^{\mu} \delta n_{\mu}-p_{i} \delta u_{i}\right) .
$$

In this expression $p_{i}$ is the momentum density, $T_{i j}$ is the spatial stress tensor, $j^{\mu}=\left(j^{0}, j^{i}\right)$ contains the number density and current and $\epsilon^{\mu}=\left(\epsilon^{0}, \epsilon^{i}\right)$ the energy density and current. $j^{i}$ is proportional to $p_{i}$ because only the combination $\delta A_{i}-\delta u_{i}$ enters the DLCQ metric, see eq. (2.12).

The first-order Weyl variation $\Delta$ of the vacuum functional nearby flat spacetime is:

$$
\Delta_{W} W=2 \sigma G_{A B} \frac{\delta W}{\delta G_{A B}}=2 \sigma\left(\delta^{i j} \frac{\delta W}{\delta\left(\delta \tilde{h}_{i j}\right)}+2 \frac{\delta W}{\delta\left(\delta n_{0}\right)}\right)=2 \sigma\left(T_{i}^{i}-2 \epsilon^{0}\right)
$$

In the rest of the paper we specialize to the $d=2$ case.

\footnotetext{
${ }^{4}$ Upper and lower spatial indices $i, j$ are raised by Kronecker delta and so are interchangeable.
} 


\subsection{The trace anomaly without Frobenius condition}

Let us consider a conformal field theory coupled to a background NC geometry. We will restrict to the parity-invariant case. A generic term inside the anomaly in $d+1$ dimensions can be written as a scalar obtained contracting the following $d+2$-dimensional tensors: the curvature $R_{A B C D}$, the null direction $n_{A}$ and the metric $G^{A B}$. We denote respectively by $N_{n}, N_{D}$ and $N_{R}$ the numbers of $n_{A}$ vectors, covariant derivatives and Riemann tensors, ${ }^{5}$ all taken with lower indices. $N_{G}$ denotes the number of metric tensors (all taken with upper indices) which are used for the contraction. The condition for a term to be a scalar is

$$
4 N_{R}+N_{n}+N_{D}=2 N_{G},
$$

while the requirement of having the correct Weyl weight in order to enter the anomaly is given by

$$
2 N_{R}+2 N_{n}-2 N_{G}=-4 .
$$

Eliminating $N_{G}$ from eqs. (2.16), (2.17), one obtains

$$
4=N_{D}+2 N_{R}-N_{n} .
$$

The number $N_{n}$ is unchanged by Weyl transformation. This observation has interesting implications: the Wess-Zumino consistency conditions of the terms with different $N_{n}$ do not mix with each other. Consequently, we can study each sector with a different value of $N_{n}$ independently.

The basis for the anomaly in the sector $N_{n}=0$ and the cohomological problem are exactly the same as the relativistic trace anomaly in $(3+1)$-dimensional space-time [31]:

$$
\mathcal{A}=a E_{4}-c W^{2}+\mathcal{A}_{\mathrm{ct}}, \quad \mathcal{A}_{\mathrm{ct}}=-\frac{2}{3} a^{\prime} \square R,
$$

where the Euler density and the Weyl tensor are calculated in the DLCQ space-time starting from the corresponding metric eq. (2.5):

$$
E_{4}=R_{A B M N}^{2}-4 R_{A B}^{2}+R^{2}, \quad W^{2}=W_{A B M N}^{2}=R_{A B M N}^{2}-2 R_{A B}^{2}+\frac{1}{3} R^{2} .
$$

In this way we immediately recognize the existence of a type $\mathrm{A}$ anomaly, the $E_{4}$ term, and of a type B anomaly, the squared Weyl tensor. The anomaly in eq. (2.19) was explicitly computed for a free scalar in [32].

The sectors with $N_{n}>0$ will be discussed in section 5 .

\subsection{The trace anomaly with Frobenius condition}

If we impose the causality condition (2.3) the structure of the anomaly drastically changes: only a finite number of non-vanishing terms are allowed by the conformal dimension. Moreover the type $A$ anomaly can be eliminated by a local counterterm and becomes schemedependent. This case was studied in [33] and in [28] with different formalisms. In this section we will summarize the results using the notation of [33].

\footnotetext{
${ }^{5}$ In the notation of [28]: $N_{T}=-N_{n}, N_{S}=N_{D}+2 N_{R}+N_{n}, N_{\epsilon}=0$.
} 
The condition that $n_{M}$ is a Killing vector for the metric gives:

$$
0=\mathcal{L}_{n}\left(G_{M N}\right)=D_{M} n_{N}+D_{N} n_{M}
$$

The condition that $n_{A}$ is null gives $D_{M} n^{M}=0$ and $n^{S} D_{S} n_{M}=0$. We define the 2-form

$$
\tilde{F}=\tilde{F}_{A B} d x^{A} \wedge d x^{B}=2 d n,
$$

which in components reads:

$$
\tilde{F}_{M N}=\partial_{M} n_{N}-\partial_{N} n_{M}=2 D_{M} n_{N} .
$$

In particular, $\tilde{F}_{-\alpha}=0$.

The causality condition $n \wedge d n=0$ implies that $d n=n \wedge w$ for some one form $w$ :

$$
\tilde{F}_{A B}=n_{[A} w_{B]}, \quad w_{A}=\left(0, w_{\alpha}\right), \quad n^{A} w_{A}=0 .
$$

Note that $w_{A}$ in eq. (2.24) is not uniquely determined; for example it could be shifted by

$$
w_{A} \rightarrow w_{A}+p n_{A},
$$

where $p$ is an arbitrary function $\left(x^{-}\right.$independent) without affecting $\tilde{F}_{A B}$.

We define:

$$
\begin{aligned}
\chi & =\frac{1}{16} G^{M N} w_{M} w_{N} . \\
\Omega_{A B} & =\frac{1}{16}\left(w_{A} w_{B}-4 D_{A} w_{B}\right), \quad \Omega=\Omega_{A B} G^{A B} .
\end{aligned}
$$

Note that $\chi$ is invariant under the shift in eq. (2.25). We can use the ambiguity in eq. (2.25) to render $\Omega_{A B}$ symmetric. The following property is useful:

$$
\Omega_{A B} n^{B}=\Omega_{B A} n^{B}=\chi n_{A} .
$$

It is convenient to introduce

$$
J=\Omega-2 \chi+\frac{R}{6},
$$

whose Weyl variation is $-2 \sigma J$.

By dimensional analysis, the anomaly can be written as a finite linear combination:

$$
\mathcal{A}_{\sigma}=\sum_{k=1}^{12} b_{k} \mathcal{A}_{\sigma}^{k}, \quad \mathcal{A}_{\sigma}^{k}=\int \sqrt{g} d^{3} x\left(\sigma A_{k}\right),
$$

where $b_{k}$ are the anomaly coefficients and

$$
\begin{aligned}
& A_{1}=D^{2} R, \quad A_{2}=R^{2}, \quad A_{3}=\chi^{2}, \\
& A_{4}=\Omega^{2}, \quad A_{5}=\chi \Omega, \quad A_{6}=\chi R, \\
& A_{7}=\Omega R, \quad A_{8}=\Omega_{A B} \Omega^{A B}, \quad A_{9}=\Omega_{A B} w^{A} w^{B}, \\
& A_{10}=w^{A} D_{A} R, \quad A_{11}=D^{2} \chi, \quad A_{12}=D^{2} \Omega .
\end{aligned}
$$


In order to determine this basis, it is important to use the following relations, which are valid only if the Frobenius condition is satisfied:

$$
\begin{aligned}
W^{2} & =12 J^{2}, \\
E_{4} & =72 \chi^{2}-4 \chi R-48 \chi \Omega+8 \Omega^{2}-8 \Omega_{A B} \Omega^{A B}, \\
\left(R_{A B}+2 \Omega_{A B}\right) w^{A} w^{B} & =8 \chi(R-6 \chi+4 \Omega), \\
\Omega_{A B}\left(R^{A B}+2 \Omega^{A B}\right) & =12 \chi^{2}+\frac{1}{2} \Omega(R+4 \Omega)-\chi(R+9 \Omega) .
\end{aligned}
$$

Imposing the Wess-Zumino consistency condition, we find that at the conformal fixed point the anomaly is of type B:

$$
\mathcal{A}=b \sigma J^{2}+\mathcal{A}_{\mathrm{ct}}
$$

where the terms in $\mathcal{A}_{\mathrm{ct}}$ are arbitrary linear combinations of

$$
\begin{aligned}
& \sigma D^{2} R, \quad \sigma D^{2}(\Omega-2 \chi), \quad \sigma\left(12 \chi^{2}-4 \chi \Omega-\frac{1}{2} \Omega_{A B} w^{A} w^{B}\right), \\
& \sigma\left(2 R \chi-2 R \Omega+\frac{w^{A} D_{A} R}{2}-6 D^{2} \chi\right), \\
& \sigma\left(-9 \chi^{2}-\Omega^{2}+6 \chi \Omega+\Omega_{A B}^{2}+\frac{\chi R}{2}\right),
\end{aligned}
$$

which can all be written as Weyl variation of local counterterms (see appendix A).

\section{Local RG without Frobenius condition and $N_{n}=0$}

The couplings $g^{i}$ are now taken as space-time dependent sources for the marginal operators $\mathcal{O}_{i}$ of the theory, e.g.:

$$
\left\langle\mathcal{O}_{i}(x)\right\rangle=\frac{\delta W}{\delta g^{i}(x)},
$$

where $W$ is the vacuum functional. The local $R G$ generator is:

$$
-\int \sqrt{g} d^{3} x\left(\Delta_{W} W+\Delta_{\beta} W\right)=\mathcal{A}
$$

where

$$
\Delta_{W} W=2 \sigma G_{A B} \frac{\delta W}{\delta G_{A B}}, \quad \Delta_{\beta} W=\sigma \beta^{k} \frac{\delta W}{\delta g_{k}},
$$

and $\beta^{k}$ denote the beta functions of each coupling: $\beta^{k}=\frac{d g^{k}}{d \log \mu}$. The anomaly $\mathcal{A}$ now includes also terms with space-time derivatives of the couplings $g^{k}$. To avoid confusion, starting from this section, the lowercase latin indices $i, j, k, \ldots$ run on the space of the couplings $g^{i}$. Moreover, we denote by $\partial_{i} f=\frac{\partial f}{\partial g^{i}}$ the derivatives of functions with respect to couplings. If the coupling $g^{i}$ has some charge $m$ under the "mass" particle number, a dependence $g^{i}=\tilde{g}^{i}\left(x^{\mu}\right) e^{i m x^{-}}$should be given in the null direction. Note that for $m=0$ the coupling $g^{i}$ can be real, while for $m \neq 0$ it must be complex. 
In the sector $N_{n}=0$, the anomaly part of the local RG equation can be formally obtained by DLCQ reduction of the relativistic local RG [6]:

$$
\mathcal{A}=\mathcal{B}+\left(D_{A} \sigma\right) \mathcal{Z}^{A}+\left(D^{2} \sigma\right) \mathcal{X}
$$

where

$$
\begin{aligned}
\mathcal{B}= & a E_{4}-c W_{A B C D}^{2}+\frac{b}{9} R^{2}+\frac{1}{3} \chi_{i}^{e} D_{A} g^{i} D^{A} R+\frac{1}{6} \chi_{i j}^{f} D_{A} g^{i} D^{A} g^{j} R+\frac{1}{2} \chi_{i j}^{g} D_{A} g^{i} D^{A} g^{j} \\
& +\frac{1}{2} \chi_{i j}^{a} D^{2} g^{i} D^{2} g^{j}+\frac{1}{2} \chi_{i j k}^{b} D_{A} g^{i} D^{A} g^{j} D^{2} g^{k}+\frac{1}{4} \chi_{i j k l}^{c} D_{A} g^{i} D^{A} g^{j} D_{B} g^{k} D^{B} g^{l} \\
\mathcal{Z}^{A}= & w_{i} E^{A B} D_{B} g^{i}+\frac{1}{3} Y_{i} R D^{A} g^{i}+S_{i j} D^{A} g^{i} D^{2} g^{j}+\frac{1}{2} T_{i j k} D^{A} g^{i} D_{B} g^{j} D^{B} g^{k} \\
\mathcal{X}= & -\frac{2 a^{\prime}}{3} R-U_{i} D^{2} g^{i}-\frac{1}{2} V_{i j} D_{B} g^{i} d^{B} g^{j} .
\end{aligned}
$$

Formally we can write exactly the same consistency conditions as in the relativistic case in $d=4$, and the same set of local counterterms can be used to shift the anomaly coefficients. A detailed analysis can be found in [6]. It is interesting to focus on the consistency conditions that give a perturbative proof of the $a$ theorem:

$$
\begin{aligned}
8 \partial_{i} a-\chi_{i j}^{g} \beta^{j} & =-\mathcal{L}_{\beta} w_{i}, \\
\chi_{i j}^{g}+2 \chi_{i j}^{a}+2 \partial_{i} \beta^{k} \chi_{k j}^{a}+\beta^{k} \chi_{k i j}^{b} & =\mathcal{L}_{\beta} S_{i j},
\end{aligned}
$$

where $\mathcal{L}_{\beta}$ is the Lie derivative in the coupling space along the direction given by the beta functions $\beta^{i}$, e.g.:

$$
\mathcal{L}_{\beta} w_{i}=\beta^{k} \partial_{k} w_{i}+\partial_{i} \beta^{k} w_{k}
$$

Then equation (3.6) can be re-written as:

$$
\beta^{i} \partial_{i} \tilde{a}=\frac{1}{8} \chi_{i j}^{g} \beta^{i} \beta^{j}, \quad \tilde{a}=a+\frac{1}{8} w_{i} \beta^{i},
$$

then the quantity $\tilde{a}$ is monotonically decreasing along the RG flow provided that $\chi_{i j}^{g}$ is a positive-definite metric. In the limit of small $\beta^{i}$ (which corresponds to leading-order in conformal perturbation theory), this is equivalent to the fact that $\chi_{i j}^{a}$ is negative-definite (see eq. (3.7)).

In the relativistic case, the positiveness of $-\chi_{i j}^{a}$ follows from the fact that it coincides, with the Zamolodchikov metric ${ }^{6}$ in an opportune class of schemes. Positivity of Zamolodchikov metric, for unitary theories, in turn follows from the positivity of the spectral density function in the Källén-Lehmann spectral representation, see [20] for a review.

The key missing ingredient for a perturbative proof of the monotonicity of $\tilde{a}$ in the non-relativistic case is the negativity of the anomaly coefficient $\chi_{i j}^{a}$. In spite of the apparent similarities with the relativistic case, the proof of the negativity of $\chi_{i j}^{a}$ does not seem to follow from a straightforward generalization of the relativistic argument. We leave this issue as a topic for further investigation.

\footnotetext{
${ }^{6}$ The Zamolodchikov metric is defined from the two-point functions of the marginal operators: $\left\langle\mathcal{O}_{i}(x) \mathcal{O}_{i}(0)\right\rangle=\frac{G_{i j}\left(g^{k}\right)}{x^{2(d+2)}}$.
} 
Another interesting consistency condition is:

$$
\beta^{k} \partial_{k}\left(4 a^{\prime}+U_{i} \beta^{i}\right)=8 b-\chi_{i j}^{a} \beta^{i} \beta^{j}
$$

The quantity $a^{\prime}$ is scheme-dependent at the fixed point; indeed if we add a counterterm

$$
\delta W=C R^{2}+\frac{1}{3} E_{i} D_{A} g^{i} D^{A} R
$$

there is the following shift in the anomaly coefficients in eq. (3.5):

$$
\delta d=2 C+\frac{1}{2} E_{i} \beta^{i}, \quad \delta b=\mathcal{L}_{\beta}(D), \quad \delta \chi_{i}^{e}=\mathcal{L}_{\beta}\left(E_{i}\right)
$$

The quantity $b$ vanishes at the conformal fixed point; then it can be written as $b=\beta^{i} \xi_{i}$ for some smooth vector $\xi_{i}$. Along a particular RG trajectory, we can write $\xi_{i}=\partial_{i} G$, and then we can use the counterterm $C=-G$ to make $b$ vanishing along an RG trajectory. In this scheme the difference $\Delta a^{\prime}=a_{U V}^{\prime}-a_{I R}^{\prime}$ corresponds to

$$
\Delta a^{\prime}=-\frac{1}{4} \int_{I R}^{U V} \chi_{i j}^{a} \frac{d g^{i}}{d t} \frac{d g^{j}}{d t} d t, \quad t=\log \mu
$$

which formally resembles the action of a free particle moving in the space of couplings. If the metric $-\chi_{i j}^{a}$ is positive-definite, then $\Delta a^{\prime}$ is always positive, even if dependent on the specific RG flow trajectory. In the relativistic case, it was conjectured $[22,23]$ that $\Delta a \leq \Delta a^{\prime}$ and that the lowest possible value of $\Delta a^{\prime}$ coincides with $\Delta a$; it is reasonable that a similar property is valid also in the non-relativistic case.

\section{Local RG with Frobenius condition}

If the Frobenius condition holds, the number of terms in the anomaly is finite. In this section we perform an analysis of the local renormalization group in the case of marginal perturbations; in this case the study of the WZ consistency conditions is formally different from the relativistic case. We know from section 2.4 that the scheme-independent content of the anomaly is just a single term of type $B$, whose coefficient is therefore unconstrained by the local RG equations. On the other hand, $T_{\mu}^{\mu}$ has several geometrical contributions which have a non-trivial RG evolution, analogously to the $a^{\prime}$ coefficients of the relativistic case. These coefficients are not universal properties of each conformal fixed point, but still may give interesting constraint on the possible RG flows.

We choose the following basis:

$$
\left(\Delta_{\sigma}^{W}+\Delta_{\sigma}^{\beta}\right) W=\int d^{3} x \sqrt{g}\left(\sigma \mathcal{B} \cdot \mathcal{R}+D_{A} \sigma \mathcal{Z}^{A}\right)
$$


where

$$
\begin{aligned}
\mathcal{B} \cdot \mathcal{R}= & \eta^{1} R^{2}+\eta^{2} \chi^{2}+\eta^{3} \Omega^{2}+\eta^{4} \chi \Omega+\eta^{5} \chi R+\eta^{6} \Omega R \\
& +\eta^{7} \Omega_{A B} \Omega^{A B}+\eta^{8} \Omega_{A B} w^{A} w^{B}+\eta^{9} w^{A} D_{A} R+\eta^{10} D^{2} R+\eta^{11} D^{2} \chi+\eta^{12} D^{2} \Omega \\
& +c_{i}^{1} D^{2} g^{i} R+c_{i}^{2} D^{2} g^{i} \chi+c_{i}^{3} D^{2} g^{i} \Omega+c_{i}^{4} D_{A} g^{i} w^{A} R+c_{i}^{5} D_{A} g^{i} w^{A} \chi+c_{i}^{6} D_{A} g^{i} w^{A} \Omega \\
& +e_{i j}^{1} D_{A} g^{i} D^{A} g^{j} R+e_{i j}^{2} D_{A} g^{i} D^{A} g^{j} \chi+e_{i j}^{3} D_{A} g^{i} D^{A} g^{j} \Omega \\
& +e_{i j}^{4} D_{A} g^{i} D_{B} g^{j} w^{A} w^{B}+e_{i j}^{5} D_{A} g^{i} D_{B} g^{j} \Omega^{A B}+e_{i j}^{6} D^{2} g^{i} D^{2} g^{j}+e_{i j}^{7} D^{2} g^{i} D^{A} g^{j} w_{A} \\
& +k_{i j m}^{1} D_{A} g^{i} D^{A} g^{j} D^{2} g^{m}+k_{i j m}^{2} D_{A} g^{i} D^{A} g^{j} D^{B} g^{m} w_{B} \\
& +q_{i j m n} D_{A} g^{i} D^{A} g^{j} D_{B} g^{m} D^{B} g^{n} \\
\mathcal{Z}^{A}= & d_{i}^{1} D_{B} g^{i} \Omega^{A B}+d_{i}^{2} D_{B} g^{i} w^{A} w^{B}+d_{i}^{3} D^{A} g^{i} R+d_{i}^{4} D^{A} g^{i} \chi \\
& +d_{i}^{5} D^{A} g^{i} \Omega+D^{A}\left(d_{i}^{6} D^{2} g^{i}\right)+d_{i}^{7} D^{2} g^{i} w^{A} \\
& +f_{i j}^{1} D^{A} g^{i} D^{2} g^{j}+D^{A}\left(f_{i j}^{2} D_{B} g^{i} D^{B} g^{j}\right)+f_{i j}^{3} D^{A} g^{i} D^{B} g^{j} w_{B} \\
& +T_{i j k} D_{B} g^{i} D^{B} g^{j} D^{A} g^{k} .
\end{aligned}
$$

The terms $\eta^{k}$ are present also in the case of constant space-time couplings and corresponds to the anomaly discussed in section 2.4. At the conformal fixed point, all the allowed terms with non-vanishing Weyl variations are variations of local counterterms, see appendix A.

Part of the WZ consistency condition give some algebraic relations which can be used to solve for $\eta^{k}, k=4, \ldots 9$ :

$$
\begin{aligned}
\eta^{4} & =\frac{1}{6}\left(-6 \beta^{i} c_{i}^{1}-\beta^{i} c_{i}^{3}+2 \beta^{i} c_{i}^{5}+8 \beta^{i} c_{i}^{6}+72 \eta^{1}-2 \eta^{2}-18 \eta^{3}\right) \\
\eta^{5} & =\frac{1}{12}\left(6 \beta^{i} c_{i}^{1}+2 \beta^{i} c_{i}^{2}+\beta^{i} c_{i}^{3}+4 \beta^{i} c_{i}^{6}-8 \beta^{i} d_{i}^{7}-72 \eta^{1}-4 \eta^{11}-8 \eta^{12}-6 \eta^{3}\right) \\
\eta^{6} & =\frac{1}{3}\left(-3 \beta^{i} c_{i}^{1}+2 \beta^{i} d_{i}^{7}+36 \eta^{1}+\eta^{11}+2 \eta^{12}\right) \\
\eta^{7} & =\frac{1}{2}\left(-6 \beta^{i} c_{i}^{1}-\beta^{i} c_{i}^{3}+72 \eta^{1}-2 \eta^{3}\right) \\
\eta^{8} & =\frac{1}{48}\left(30 \beta^{i} c_{i}^{1}+5 \beta^{i} c_{i}^{3}+2 \beta^{i} c_{i}^{5}-4 \beta^{i} c_{i}^{6}-360 \eta^{1}-2 \eta^{2}+18 \eta^{3}\right) \\
\eta^{9} & =\frac{1}{12}\left(-2 \beta^{i} d_{i}^{7}-\eta^{11}-2 \eta^{12}\right)
\end{aligned}
$$

The remaining consistency conditions are listed in appendix B. Starting from these, we can identify some possible candidates for monotonicity properties. Let us introduce:

$$
\begin{aligned}
& \tilde{A}_{1}=-2 \eta^{12}+12 \eta^{10}-2 \beta^{k} d_{k}^{6} \\
& \tilde{A}_{2}=48 \eta^{1}+\frac{2}{3} \eta^{2}-4 \eta^{3}+\beta^{k}\left(-4 c_{k}^{1}-\frac{2}{3} c_{k}^{3}-\frac{2}{3} c_{k}^{5}+\frac{4}{3} c_{k}^{6}-d_{k}^{1}-16 d_{k}^{2}\right) \\
& \tilde{A}_{3}=-72 \eta^{1}+2 \eta^{3}-4 \eta^{11}-8 \eta^{12}+\beta^{k}\left(6 c_{k}^{1}+c_{k}^{3}-d_{k}^{1}-8 d_{k}^{7}\right) \\
& \tilde{A}_{4}=\eta^{11}+2 \eta^{12}-6 \eta^{10}+\beta^{k}\left(3 d_{k}^{3}-3 c_{k}^{1}+2 d_{k}^{7}\right) .
\end{aligned}
$$


From eq. (B.1), (B.2), (B.3), (B.4) we find the following relations:

$$
\begin{aligned}
\beta^{i} \partial_{i} \tilde{A}_{1}+\beta^{i}\left(12 c_{i}^{1}-2 c_{i}^{3}\right)-4 e_{i j}^{6} \beta^{i} \beta^{j} & =0, \\
\beta^{i} \partial_{i} \tilde{A}_{2}+\left(32 e_{i j}^{4}+2 e_{i j}^{5}\right) \beta^{j} \beta^{i}-\left(8 c_{i}^{5}+16 c_{i}^{6}\right) \beta^{i} & =0, \\
\beta^{i} \partial_{i} \tilde{A}_{3}+\beta^{i}\left(-2 c_{i}^{2}-4 c_{i}^{3}-24 c_{i}^{4}+4 c_{i}^{6}\right)+\left(2 e_{i j}^{5}+8 e_{(i j)}^{7}\right) \beta^{i} \beta^{j} & =0, \\
\beta^{i} \partial_{i} \tilde{A}_{4}+\beta^{i}\left(12 c_{i}^{4}-6 c_{i}^{1}-3 c_{l}^{1}\left(\partial_{i} \beta^{l}\right)+3\left(\partial_{i} c_{l}^{1}\right) \beta^{l}\right)-6 e_{i j}^{1} \beta^{i} \beta^{j} & =0 .
\end{aligned}
$$

Each of the previous equations has a structure which is reminiscent of the relativistic equation for $a^{\prime}$, see eq. (3.10). Note in fact that the terms proportional to $c_{i}^{k}$ can be shifted using the counterterms in appendix A and set to zero along a RG trajectory. Consequently, we may consider linear combinations of the four previous quantities $\tilde{A}_{k}$ as candidates for monotonicity theorems. In particular, $e_{i j}^{6}$ is the analog of the $\frac{1}{2} \chi_{i j}^{a}$ of the previous section because it is the coefficient of the $\sigma D^{2} g^{i} D^{2} g^{j}$ term of the anomaly, so $\tilde{A}_{1}$ is identified with the $a^{\prime}$ term.

\section{The $N_{n}>0$ sectors}

In the absence of Frobenius condition (2.3), the number of possible terms in the anomaly basis is infinite. Each term can be formed solely by a combination of vectors $n_{A}$, covariant derivatives $D_{A}$, curvature tensors $R_{A B C D}$ and metric tensors $G^{A B}$. These terms can be conveniently organized and classified. A convenient way is to introduce an integer number $N_{n}$ labelling the number of DLCQ vectors $n_{A}$ entering the term. This partition splits the infinite terms into infinite sectors identified by the value of $N_{n}$. This has a double advantage: a) Since a Weyl transformation does not modify the value of $N_{n}$, anomalies can be studied separately in each sector $N_{n}$; b) Although the number of sectors is infinite, the possible anomaly terms are finite within a given sector.

Inside each sector, the following relations limit the number of possible terms:

I the internal symmetries of the curvature tensors,

II the Bianchi identity

$$
D_{E} R_{A B C D}+D_{C} R_{A B D E}+D_{D} R_{A B E C}=0
$$

III since $n^{A}$ generates an isometry, the following Lie derivatives vanish

$$
\begin{aligned}
\mathcal{L}_{n}\left(G_{A B}\right) & =0, \\
\mathcal{L}_{n}(R) & =0, \\
\mathcal{L}_{n}\left(R_{A B}\right) & =0, \\
\mathcal{L}_{n}\left(R_{A B C D}\right) & =0 .
\end{aligned}
$$

Let us fix a value of $N_{n}$. Then, a dimensional analysis constraints the number $N_{R}$ of curvature tensors entering the anomaly term to the following

$$
0 \leq N_{R} \leq \begin{cases}\frac{1}{2}\left(N_{n}+3\right) & \text { if } N_{n} \text { odd } \\ \frac{1}{2}\left(N_{n}+4\right) & \text { if } N_{n} \text { even }\end{cases}
$$


This splits the sector $N_{n}$ into distinct subsectors labelled by $N_{R}$. Once $N_{R}$ is fixed, the number of covariant derivatives $N_{D}$ is also fixed according to eq. (2.18). We will often make use of the following commutation relation to move terms into different subsectors $N_{R}$

$$
\begin{aligned}
{\left[D_{M}, D_{N}\right] X_{B_{1} \ldots B_{l}}^{A_{1} \ldots A_{k}}=} & +R_{P M N}^{A_{1}} X_{P B_{1} \ldots B_{l}}^{P A_{2} \ldots A_{k}}+\ldots \\
& -R_{B_{1} M N}^{P} X_{P B_{2} \ldots B_{l}}^{A_{1} \ldots A_{k}}-\ldots
\end{aligned}
$$

The following systematic procedure can be applied to find a basis of terms entering the anomaly for each separate sector $N_{n}$ :

a) Find all the possible subsectors with $N_{R}$ going from 0 to the value (5.3).

b) Start from $N_{R}=0$; use repeatedly eq. (5.4) to move terms of this subsector to subsectors with higher $N_{R}$.

c) Within the remaining terms, apply the relations I-III to restrict the numbers of possible terms in the anomaly basis.

d) Increase $N_{R}$ by one and repeat the points b) and c).

e) The final basis will be the union of the basis obtained for each subsectors.

\subsection{The sector $N_{n}=1$}

We apply the procedure considering the simplest non trivial sector, i.e. $N_{n}=1$. According to (5.3), we have three subsectors $N_{R}=0,1,2$. Without taking into account geometrical constraints, such as eqs. (5.1), (5.2), the number of possible terms forming the basis of the anomaly is 86 . The procedure above is a systematic way to select the linearly independent terms; although it is simple in principle, it is quite lengthy and non-trivial in practical uses; it selects just 3 terms out of 86 . The result is summarized in table 1, and the basis is given by the bold terms.

The result is the basis:

$$
\begin{aligned}
& B_{1}=\left(n^{A} R_{A B}\right) D^{B} R \\
& B_{2}=\left(D_{C} R_{A B}\right) n^{A} R^{B C} \\
& B_{3}=\left(n^{D} R_{A B C D}\right) D^{B} R^{A C},
\end{aligned}
$$

and the anomaly:

$$
\mathcal{A} \supset \int d^{3} x \sqrt{g} \sigma \sum_{k=1}^{3} f_{k} B_{k} .
$$

\subsection{Cohomological problem of the $N_{n}=1$ sector}

It is possible to show that none of the three terms in the basis can be written as a Weyl variation of any combination of the remaining ones. Consequently, there are not counterterms (i.e. scheme dependent terms) in the anomaly. 


\begin{tabular}{|c|c|c|c|}
\hline Subsector & Possible terms & & \\
\hline$N_{R}=0$ & $D_{A} D_{B} D_{C} D^{C} D^{B} n^{A}$, & $D_{A} D_{C} D_{B} D^{C} D^{B} n^{A}$, & $D_{A} D_{C} D^{C} D_{B} D^{B} n^{A}$ \\
\hline \multirow[t]{4}{*}{$N_{D}=5$} & $D_{B} D_{A} D_{C} D^{C} D^{B} n^{A}$ & $D_{B} D_{C} D_{A} D^{C} D^{B} n^{A}$ & $D_{B} D_{C} D^{C} D_{A} D^{B} n^{A}$ \\
\hline & $D_{B} D_{C} D^{C} D^{B} D_{A} n^{A}$, & $D_{C} D_{A} D_{B} D^{C} D^{B} n^{A}$, & $D_{C} D_{A} D^{C} D_{B} D^{B} n^{A}$ \\
\hline & $D_{C} D_{B} D_{A} D^{C} D^{B} n^{A}$, & $D_{C} D_{B} D^{C} D_{A} D^{B} n^{A}$, & $D_{C} D_{B} D^{C} D^{B} D_{A} n^{A}$, \\
\hline & $D_{C} D^{C} D_{A} D_{B} D^{B} n^{A}$ & $D_{C} D^{C} D_{B} D_{A} D^{B} n^{A}$, & $D_{C} D^{C} D_{B} D^{B} D_{A} n^{A}$. \\
\hline \multirow{16}{*}{$\begin{array}{l}N_{R}=1 \\
N_{D}=3\end{array}$} & $R\left(D_{A} D_{B} D^{B} n^{A}\right)$ & $R\left(D_{B} D_{A} D^{B} n^{A}\right)$ & $R\left(D_{B} D^{B} D_{A} n^{A}\right)$, \\
\hline & $R_{B C}\left(D_{A} D^{C} D^{B} n^{A}\right)$ & $R_{A C}\left(D_{B} D^{C} D^{B} n^{A}\right)$ & $R_{A B}\left(D_{C} D^{C} D^{B} n^{A}\right)$ \\
\hline & $R_{B C}\left(D^{C} D_{A} D^{B} n^{A}\right)$ & $R_{A C}\left(D^{C} D_{B} D^{B} n^{A}\right)$ & $R_{B C}\left(D^{C} D^{B} D_{A} n^{A}\right)$ \\
\hline & $R_{A B C D}\left(D^{D} D^{C} D^{B} n^{A}\right)$, & $R_{A C B D}\left(D^{D} D^{C} D^{B} n^{A}\right)$ & $R_{A D B C}\left(D^{D} D^{C} D^{B} n^{A}\right)$. \\
\hline & $\left(D_{A} D_{B} n^{B}\right) D^{A} R$ & $\left(D_{B} D_{A} D_{A} n^{B}\right) D^{A} R$ & $\left(D_{B} D^{B} n_{A}\right) D_{A} R$ \\
\hline & $\left(D_{B} D^{B} n^{A}\right) D^{C} R_{A C}$, & $\left(D_{A} D^{B} n^{A}\right) D^{C} R_{B C}$ & $\left(D^{B} D_{A} n^{A}\right) D^{C} R_{B C}$, \\
\hline & $\left(D_{C} D_{B} n_{A}\right) D^{A} R^{B C}$ & $\left(D_{C} D_{B} n_{A}\right) D^{B} R_{A C}$ & $\left(D_{C} D_{B} n_{A}\right) D^{C} R^{A B}$ \\
\hline & $\left(D_{C} D_{B} n_{A}\right) D_{D} R^{A B C D}$ & $\left(D_{C} D_{B} n_{A}\right) D_{D} R^{A C B D}$ & $\left(D_{C} D_{B} n_{A}\right) D_{D} R^{A D B C}$. \\
\hline & $\left(D_{A} n^{A}\right) D_{B} D^{B} R$ & $\left(D_{B} n_{A}\right) D^{A} D^{B} R$ & $\left(D_{B} n_{A}\right) D_{B} D^{A} R$ \\
\hline & $\left(D_{B} n^{A}\right) D_{A} D_{C} R^{B C}$, & $\left(D^{B} n_{A}\right) D_{B} D_{C} R^{A C}$ & $\left(D_{B} n^{A}\right) D_{C} D_{A} R^{B C}$ \\
\hline & $\left(D^{B} n_{A}\right) D_{C} D_{B} R^{A C}$ & $\left(D_{A} n^{A}\right) D_{C} D_{B} R^{B C}$ & $\left(D_{B} n_{A}\right) D_{C} D^{C} R^{A B}$ \\
\hline & $\left(D_{B} n_{A}\right) D_{C} D_{D} R^{A B C D}$ & $\left(D_{B} n_{A}\right) D_{C} D_{D} R^{A C B D}$ & $\left(D_{B} n_{A}\right) D_{C} D_{D} R^{A D B C}$. \\
\hline & $n^{A}\left(D_{A} D_{B} D^{B} R\right)$ & $n_{A}\left(D_{B} D^{A} D^{B} R\right)$ & $n_{A}\left(D_{B} D^{B} D^{A} R\right)$ \\
\hline & $n^{A}\left(D_{A} D_{C} D_{B} R^{B C}\right)$, & $n_{A}\left(D_{B} D_{C} D^{C} R^{A B}\right)$ & $n^{A}\left(D_{C} D_{A} D_{B} R^{B C}\right)$ \\
\hline & $n^{A}\left(D_{C} D_{B} D_{A} R^{B C}\right)$ & $n_{A}\left(D_{C} D_{B} D^{C} R^{A B}\right)$ & $n_{A}\left(D_{C} D^{C} D_{B} R^{A B}\right)$ \\
\hline & $n^{A}\left(D_{B} D_{D} D_{C} R^{A B C D}\right)$, & $n^{A}\left(D_{B} D_{D} D_{C} R^{A C B D}\right)$ & $n^{A}\left(D_{B} D_{D} D_{C} R^{A D B C}\right)$. \\
\hline \multirow{10}{*}{$\begin{array}{l}N_{R}=2 \\
N_{D}=1\end{array}$} & $\left(D_{A} n^{A}\right) R^{2}$ & $\left(D_{B} n_{A}\right) R R^{A B}$ & $\left(D_{A} n^{A}\right) R_{B C} R^{B C}$ \\
\hline & $\left(D_{B} n_{A}\right) R_{C}^{A} R^{B C}$ & $\left(D_{B} n_{A}\right) R_{C D} R^{A C B D}$ & \\
\hline & $\left(D_{A} n^{A}\right) R^{B C D E} R^{B C D E}$, & $\left(D_{A} n^{A}\right) R^{B C D E} R^{B D C E}$, & \\
\hline & $\left(D_{B} n_{A}\right) R^{B C D E} R_{C D E}^{A}$, & $\left(D_{B} n_{A}\right) R^{B D C E} R_{C D E}^{A}$. & \\
\hline & $n^{A} R\left(D_{A} R\right)$ & $n^{A} R_{A B}\left(D^{B} R\right)$ & $n_{A} R\left(D_{B} R^{A B}\right)$ \\
\hline & $n_{A} R_{B C}\left(D^{C} R^{A B}\right)$ & $n_{A} R^{A B}\left(D^{C} R_{B C}\right)$, & $n_{A} R_{B C}\left(D^{A} R^{B C}\right)$, \\
\hline & $n_{A} R_{B C}\left(D_{D} R^{A B C D}\right)$ & $n^{A} R_{A B C D}\left(D^{D} R^{B C}\right)$ & \\
\hline & $n^{A} R_{A B C D}\left(D_{E} R^{B C D E}\right)$, & $n^{A} R_{A B C D}\left(D_{E} R^{B E C D}\right)$ & \\
\hline & $n_{A} R_{B C D E}\left(D^{A} R^{B C D E}\right)$, & $n_{A} R_{B C D E}\left(D^{A} R^{B D C E}\right)$, & \\
\hline & $n_{A} R_{B C D E}\left(D^{C} R^{A B D E}\right)$, & $n_{A} R_{B C D E}\left(D^{E} R^{A B C D}\right)$. & \\
\hline
\end{tabular}

Table 1. List of all the 86 scalars with Weyl weight -4 built with 1 vector $n_{A}$. The bold terms compose the final basis for the anomaly. 
We can now study the cohomological problem by finding the commutator of two Weyl variations

$$
\Delta_{\sigma_{1} \sigma_{2}}^{\mathrm{WZ}} \mathcal{B}_{k}=\delta_{\sigma_{1}(x)} \int d^{3} x \sqrt{g} B_{k} \sigma_{2}(y)-\delta_{\sigma_{2}(x)} \int d^{3} x \sqrt{g} B_{k} \sigma_{1}(y),
$$

for all the terms in the basis, $k=1,2,3$.

Using integration by parts, eq. (5.7) can be written as a linear combination of 9 independent expressions $W_{j}$ :

$$
\Delta_{\sigma_{1} \sigma_{2}}^{\mathrm{WZ}} \mathcal{B}_{k}=\int d^{3} x \sqrt{g}\left(\sum_{k=1}^{9} M^{k j} W_{j}\right) .
$$

The null space of the matrix $M^{k j}$ corresponds to the consistent combination entering the anomaly.

The nine independent expressions are:

$$
\begin{array}{ll}
W_{1}=\left(\sigma_{[1} D_{A} \sigma_{2]}\right) n_{B} D^{A} D^{B} R, & W_{2}=\left(\sigma_{[1} D_{A} \sigma_{2]}\right) R n_{B} R^{A B}, \\
W_{3}=\left(\sigma_{[1} D_{A} \sigma_{2]}\right) n_{B} R^{A C} R_{B C}, & W_{4}=\left(\sigma_{[1} D_{A} \sigma_{2]}\right) R_{B C} n_{D} R^{A B C D}, \\
W_{5}=\left(\sigma_{[1} D_{A} \sigma_{2]}\right)\left(D_{B} n_{C}\right) D^{C} R^{A B}, & W_{6}=\left(D_{A} \sigma_{[1} D^{2} \sigma_{2]}\right) n_{B} R^{A B}, \\
W_{7}=\left(D_{A} \sigma_{[1} D_{B} \sigma_{2]}\right) n_{C} D^{A} R^{B C}, & W_{8}=\left(D_{A} \sigma_{[1} D_{B} \sigma_{2]}\right)\left(D_{C} n^{B}\right) R^{A C}, \\
W_{9}=\left(D_{A} \sigma_{[1} D_{B} D_{C} \sigma_{2]}\right) n_{D} R^{A B C D} . &
\end{array}
$$

The transpose of the matrix $M^{k j}$ is:

$$
\left(M^{t}\right)^{m k}=\left(\begin{array}{ccc}
2 & -1 & \frac{3}{2} \\
-2 & 1 & 0 \\
0 & 0 & -3 \\
0 & 0 & 3 \\
0 & 0 & -3 \\
6 & 1 & 1 \\
0 & -2 & -1 \\
0 & -4 & 4 \\
0 & 0 & -2
\end{array}\right) .
$$

The null space of this matrix has dimension 0 and so the coefficients $f_{k}$ in eq. (5.6) must vanish. There is not any consistent term which satisfies the Wess-Zumino conditions in the $N_{n}=1$ sector.

\subsection{Sectors with higher $N_{n}$}

The problem of the higher $N_{n}$ sectors is obviously the proliferation of terms in the basis. Although the procedure is clear in principle, the problem is intractable on the practical side unless faced with suitable computer software. This goes beyond the purpose of the present paper. In principle, the possibility that other type A anomalies exists in higher $N_{n}$ sectors 
cannot be discarded. What is for sure is that an infinite set of type B anomalies can be found, suitably combining Weyl tensors with $n$ vectors [31]. For instance, the combination

$$
W_{M N P Q} W^{M N P S} W_{A S B}^{Q} n^{A} n^{B}
$$

is non-vanishing, belongs to the sector $N_{n}=2$, has the correct Weyl weight and has vanishing Weyl variation. Similar combinations can be built for any even value of $N_{n}$, because by dimensional analysis one Weyl tensor balances $2 n_{A}$ vectors, and the number of type B anomalies is infinite.

\section{Conclusions}

In this paper we initiated the study of the local renormalization group formalism in the case of a non-relativistic theory with boost invariance in $d=2$ spatial dimensions and nearby a fixed point with dynamical exponent $z=2$.

If we do not impose the Frobenius condition, the trace anomaly and the local RG contain an infinite number of terms, organized in an infinite number of sectors with decoupled equations. In each sector there is only a finite number of terms; in particular, in the simplest sector $N_{n}=0$ the WZ consistency conditions are formally the same as in the relativistic case in 4 space-time dimensions. Moreover we investigated the structure of the trace anomaly in the sector $N_{n}=1$ and we found that it is vanishing at the conformal fixed point.

If the Frobenius condition is imposed, the structure of the trace anomaly is much simpler; only a finite number of terms are allowed by dimensional analysis. Moreover, all the quantities that are constrained by the local RG equation are scheme-dependent at the fixed point. However, it could be that they still give useful constraints on the RG flow, similarly to the $a^{\prime}$ conjecture in the relativistic case [22, 23]. Indeed, we have found four scheme-dependent quantities $\tilde{A}_{1} \ldots \tilde{A}_{4}$ whose evolution is described by an equation which is similar to the one for $a^{\prime}$ in the relativistic case. An analogous study was performed in [29] in the case of non-relativistic theories without boost invariance.

Several directions deserve further investigation:

- The proof of a conjectured $a$-theorem in the $N_{n}=0$ sector of the anomaly without Frobenius condition is reduced to the positivity of a quadratic form in the coupling space $\chi_{i j}^{g}$. In spite of the formal similarities with the relativistic case, the proof does not seem to be trivial. It would be interesting to check this issue in examples and eventually to search for a proof.

- The anomaly sectors without Frobenius condition may contain other type $A$ anomalies. Unfortunately the number of terms quickly proliferates as we increase $N_{n}$; a more efficient strategy is needed for a systematic analysis.

- We studied in detail just the $z=2, d=2$ case. The DLCQ reduction still works for generic $z$, provided that one assigns different Weyl weights to different components of extra dimensional tensors, see appendix A of [33]. Anomalies can arise only when 
$d+z$ is an even number; the case that we considered is one of the simplest and most commonly used in condensed matter applications. It would be interesting to study other combinations $(d, z)$.

- The scheme-dependent quantities $\tilde{A}_{1} \ldots \tilde{A}_{4}$ are constrained by the local RG group equation in the Frobenius case. The monotonicity of these quantities is another open question.

- In the conjecture proposed in [22, 23], the minimum of the quantity $\Delta a^{\prime}$ along all the possible RG flows is related to the difference $\Delta a$, which is a independent on the RG trajectory. It would interesting to find scheme-independent quantities related to $\tilde{A}_{1} \ldots \tilde{A}_{4}$ in a similar way.

\section{Acknowledgments}

We are grateful to Diego Redigolo for a useful discussion.

\section{A Local counterterms in the Frobenius case}

Here we will consider the following local counterterms in the vacuum functional $W$ :

$$
W \rightarrow W+\int \sqrt{g} d^{3} x\left[K_{1} R^{2}+K_{2}(\Omega-2 \chi)^{2}+K_{3} \chi^{2}+K_{4} \Omega^{2}+K_{5} \chi R+K_{6} J^{2}\right] .
$$

We use the Weyl variations in table 2 .

The variation of each term induces a shift in the anomaly coefficients in eq. (4.1). The local RG equations in eq. (4.4) and in appendix B are invariant under these shifts; this provides several non-trivial cross-checks of our calculations. The list of the shifts induced by each term is:

1. Counterterm $K_{1}$ :

$$
\begin{gathered}
\Delta\left(K_{1} R^{2}\right)=\sigma \beta^{i} \partial_{i} K_{1} R^{2}-12 K_{1}\left(D^{2} \sigma\right) R, \\
\delta \eta^{1}=\beta^{i} \partial_{i} K_{1}, \quad \delta d_{i}^{3}=24 \partial_{i} K_{1}, \quad \delta \eta^{10}=-12 K_{1}, \\
\delta c_{i}^{1}=12 \partial_{i} K_{1}, \quad \delta e_{i j}^{1}=12 \partial_{i j} K_{1} .
\end{gathered}
$$

2. Countertem $K_{2}$ :

$$
\begin{array}{rlrl}
\Delta\left(K_{2}(\Omega-2 \chi)^{2}\right) & =\sigma \beta^{i} \partial_{i} K_{2}(\Omega-2 \chi)^{2}+2 K_{2}\left(D^{2} \sigma\right)(\Omega-2 \chi) \\
\delta \eta^{2}=4 \mathcal{L}\left(K_{2}\right), & \delta \eta^{3}=\mathcal{L}\left(K_{2}\right), & \delta \eta^{4}=-4 \mathcal{L}\left(K_{2}\right), & \delta \eta^{11}=-4 K_{2}, \\
\delta \eta^{12}=2 K_{2}, & \delta c_{i}^{2}=4 \partial_{i} K_{2}, & \delta c_{i}^{3}=-2 \partial_{i} K_{2}, & \delta d_{i}^{4}=8 \partial_{i} K_{2}, \\
\delta d_{i}^{5}=-4 \partial_{i} K_{2}, & \delta e_{i j}^{2}=4 \partial_{i j} K_{2}, & \delta e_{i j}^{3}=-2 \partial_{i j} K_{2} .
\end{array}
$$




\begin{tabular}{|l|l|}
\hline Term & Weyl variation \\
\hline$R$ & $-2 \sigma R-6 D^{2} \sigma$ \\
$\chi$ & $-2 \sigma \chi-\frac{1}{2} w^{A} \partial_{A} \sigma$ \\
$\Omega$ & $-2 \sigma \Omega-w^{A} \partial_{A} \sigma+D^{2} \sigma$ \\
\hline$w_{A}$ & $-4 D_{A} \sigma$ \\
$w^{A}$ & $-2 \sigma w^{A}-4 D^{A} \sigma$ \\
\hline$R_{M N}$ & $-2 D_{M} D_{N} \sigma-G_{M N} D^{2} \sigma$ \\
$\Omega_{C B}$ & $D_{B} D_{C} \sigma-\frac{1}{4} G_{B C} w^{K} D_{K} \sigma$ \\
\hline$R^{2}$ & $-4 \sigma R^{2}-12 R D^{2} \sigma$ \\
$(\Omega-2 \chi)^{2}$ & $-4 \sigma(\Omega-2 \chi)^{2}+2(\Omega-2 \chi) D^{2} \sigma$ \\
$\chi^{2}$ & $-4 \sigma \chi^{2}-\chi w^{A} D_{A} \sigma$ \\
$\Omega^{2}$ & $-4 \sigma \Omega^{2}-2 \Omega w^{A} D_{A} \sigma+2 \Omega D^{2} \sigma$ \\
$\chi \Omega$ & $-4 \sigma \chi \Omega-\left(\chi+\frac{1}{2} \Omega\right) w^{A} D_{A} \sigma+\chi D^{2} \sigma$ \\
$\chi R$ & $-4 \sigma \chi R-\frac{1}{2} R w^{A} D_{A} \sigma-6 \chi D^{2} \sigma$ \\
$\Omega R$ & $-4 \sigma \Omega R-R w^{A} D_{A} \sigma+(R-6 \Omega) D^{2} \sigma$ \\
$\Omega_{A B} \Omega^{A B}$ & $-4 \sigma \Omega_{A B} \Omega^{A B}+2 \Omega_{A B} D^{A} D^{B} \sigma-\frac{1}{2} \Omega w^{A} \partial_{A} \sigma$ \\
$\Omega_{A B} w^{A} w^{B}$ & $-4 \sigma \Omega_{A B} w^{A} w^{B}+w^{A} w^{B} D_{A} D_{B} \sigma-4 \chi w^{A} D_{A} \sigma-8 \Omega_{A B} w^{A} D^{B} \sigma$ \\
$w^{A} D_{A} R$ & $-4 \sigma w^{A} D_{A} R-4 D^{A} \sigma D_{A} R-2 R w^{A} D_{A} \sigma-6 w^{A} D_{A} D^{2} \sigma$ \\
\hline
\end{tabular}

Table 2. Weyl variation of several terms.

3. Counterterm $K_{3}$, modulo integration by parts:

$$
\begin{aligned}
& \Delta\left(K_{3} \chi^{2}\right)= \sigma \beta^{i} \partial_{i} K_{3} \chi^{2}-K_{3} \chi w^{A} D_{A} \sigma \\
&= \sigma \beta^{i} \partial_{i} K_{3} \chi^{2}+\sigma K_{3}\left(12 \chi^{2}-4 \chi \Omega-\frac{1}{2} \Omega_{A B} w^{A} w^{B}\right) \\
&+\sigma \partial_{i} K_{3} \chi w^{A} D_{A} g^{i} \\
& \delta \eta^{2}=\mathcal{L}\left(K_{3}\right)+12 K_{3}, \quad \delta \eta^{4}=-4 K_{3}, \quad \delta \eta^{8}=-\frac{1}{2} K_{3}, \quad \delta c_{i}^{5}=\partial_{i} K_{3} .
\end{aligned}
$$

4. Counteterm $K_{4}$, modulo integ by parts:

$$
\begin{aligned}
\Delta\left(K_{4} \Omega^{2}\right)= & \sigma \mathcal{L}\left(K_{4}\right) \Omega^{2}+2 K_{4} \Omega D^{2} \sigma-2 K_{4} \Omega w^{A} D_{A} \sigma \\
= & \sigma\left(\mathcal{L}\left(K_{4}\right)-8 K_{4}\right) \Omega^{2}+2 K_{4} \Omega D^{2} \sigma+\sigma 2 \partial_{i} K_{4} \Omega w^{A} D_{A} g^{i} \\
& +\sigma K_{4}\left(-3 \Omega_{A B} w^{A} w^{B}+8 \Omega_{A B}^{2}-4 D^{2} \chi+4 \chi R+24 \chi \Omega\right),
\end{aligned}
$$


5. Counterterm $K_{5}$ :

$$
\begin{aligned}
& \Delta\left(K_{5} \chi R\right)= \sigma \mathcal{L}\left(K_{5}\right) \chi R-\frac{K_{5}}{2} R w^{A} D_{A} \sigma-6 K_{5} \chi D^{2} \sigma \\
&= \sigma \mathcal{L}\left(K_{5}\right) R \chi+K_{5}\left(2 \sigma R \chi-2 \sigma R \Omega-6 \chi D^{2} \sigma+\frac{1}{2} \sigma w^{A} D_{A} R\right) \\
&+\frac{\sigma}{2} \partial_{i} K_{5} R w^{A} D_{A} g^{i} \\
& \delta \eta^{5}=\mathcal{L}\left(K_{5}\right)+ 2 K_{5}, \quad \delta \eta^{6}=-2 K_{5}, \quad \delta \eta^{9}=\frac{1}{2} K_{5}, \quad \delta \eta^{11}=-6 K_{5} \\
& \delta c_{i}^{2}=6 \partial_{i} K_{5}, \quad \delta c_{i}^{4}=\frac{1}{2} \partial_{i} K_{5}, \quad \delta d_{i}^{4}=12 \partial_{i} K_{5}, \quad \delta e_{i j}^{2}=6 \partial_{i j} K_{5} .
\end{aligned}
$$

6. Counterterm $K_{6}$ :

$$
\begin{array}{rlrl}
\Delta\left(F J^{2}\right) & =\Delta\left(F(\Omega-2 \chi+R / 6)^{2}\right)=\mathcal{L}(F)(\Omega-2 \chi & +R / 6)^{2}, \\
\delta \eta^{1} & =\frac{1}{36} \mathcal{L}(F), & \delta \eta^{2}=4 \mathcal{L}(F), & \delta \eta^{3}=\mathcal{L}(F), \\
\delta \eta^{4}=-4 \mathcal{L}(F), & \delta \eta^{5}=-\frac{2}{3} \mathcal{L}(F), & \delta \eta^{6}=\frac{1}{3} \mathcal{L}(F) .
\end{array}
$$

\section{B Consistency conditions with Frobenius conditions}

The WZ consistency conditions generate the algebraic constraint in eq. (4.4) and the following system of differential equations:

$$
\begin{gathered}
\partial_{i}\left(-2 \eta^{12}+12 \eta^{10}\right)+12 c_{i}^{1}-2 \mathcal{L}\left(d_{i}^{6}\right)-4 e_{i j}^{6} \beta^{j}-2 c_{i}^{3}=0 \\
\partial_{i}\left(48 \eta^{1}+\frac{2}{3} \eta^{2}-4 \eta^{3}-\beta^{k}\left(4 c_{k}^{1}+\frac{2}{3} c_{k}^{3}+\frac{2}{3} c_{k}^{5}-\frac{4}{3} c_{k}^{6}\right)\right)-\mathcal{L}\left(d_{i}^{1}+16 d_{i}^{2}\right) \\
+\left(32 e_{i j}^{4}+2 e_{i j}^{5}\right) \beta^{j}-8\left(c_{i}^{5}+2 c_{i}^{6}\right)=0 \\
\partial_{i}\left(-72 \eta^{1}+2 \eta^{3}-4 \eta^{11}-8 \eta^{12}\right)+\partial_{i}\left(\beta^{k}\left(6 c_{k}^{1}+c_{k}^{3}-4 d_{k}^{7}\right)\right)-2 c_{i}^{2}-4 c_{i}^{3} \\
-24 c_{i}^{4}+4 c_{i}^{6}+\mathcal{L}\left(-d_{i}^{1}-4 d_{i}^{7}\right)+\left(2 e_{i j}^{5}+8 e_{(i j)}^{7}\right) \beta^{j}
\end{gathered}
$$




$$
\begin{gathered}
-6 \partial_{j} \partial_{i} \eta^{9}+6 \partial_{i} c_{j}^{4}-\partial_{i} c_{j}^{6}-8 e_{i j}^{4}+2 e_{i j}^{7}-\left(\partial_{i} e_{l j}^{7}\right) \beta^{l}+e_{l j}^{7}\left(\partial_{i} \beta^{l}\right)-\beta^{l} \partial_{l} f_{i j}^{3} \\
+\left(f_{l j}^{3}+f_{j l}^{3}\right) \partial_{i} \beta^{l}+2 k_{l i j}^{2} \beta^{l}-\frac{1}{2} \partial_{i} \partial_{j} \eta^{11}-\partial_{i} \partial_{j} \eta^{12}-\frac{1}{2} \partial_{j} c_{i}^{2}-\partial_{j} c_{i}^{3}-\partial_{j} \mathcal{L}\left(d_{i}^{7}\right)+\partial_{j} e_{i k}^{7} \beta^{k}=0, \\
-d_{l}^{7}\left(\partial_{i} \partial_{j} \beta^{l}\right)-\frac{1}{2} e_{i j}^{2}-e_{i j}^{3}-\frac{1}{4} e_{i j}^{5}+k_{i j l}^{2} \beta^{l}+\frac{1}{2} \partial_{j} c_{i}^{2}+\partial_{j} c_{i}^{3}+\partial_{j} \mathcal{L}\left(d_{i}^{7}\right)-\partial_{j} e_{i k}^{7} \beta^{k}=0, \\
\partial_{i} \partial_{j}\left(6 \eta^{10}-\eta^{12}\right)+6 \partial_{j} c_{i}^{1}-\partial_{j} c_{i}^{3}-\left(\partial_{j} \beta^{l}\right)\left(\partial_{l} d_{i}^{6}\right)-\beta^{l}\left(\partial_{l} \partial_{j} d_{i}^{6}\right)-\left(\partial_{i} \beta^{l}\right)\left(\partial_{j} d_{l}^{6}\right)-d_{l}^{6} \partial_{i} \partial_{j} \beta^{l} \\
-e_{i j}^{5}+2 e_{i l}^{6}\left(\partial_{j} \beta^{l}\right)+4 e_{i j}^{6}-2\left(\partial_{j} e_{i l}^{6}\right) \beta^{l}-4 e_{i j}^{7}-\beta^{l} \partial_{l} f_{j i}^{1}-f_{l i}^{1}\left(\partial_{j} \beta^{l}\right)-f_{j l}^{1}\left(\partial_{i} \beta^{l}\right)+2 k_{l j i}^{1} \beta^{l}=0 \\
12 e_{i j}^{1}-2 e_{j i}^{3}-e_{i j}^{5}-2 \beta^{l} \partial_{l} f_{i j}^{2}-2 f_{i l}^{2}\left(\partial_{j} \beta^{l}\right)-2 f_{j l}^{2}\left(\partial_{i} \beta^{l}\right)-2 k_{i j m}^{1} \beta^{m} \\
-2 d_{l}^{6}\left(\partial_{i} \partial_{j} \beta^{l}\right)+\partial_{i j}\left(12 \eta^{10}-2 \eta^{12}\right)=0 \\
-\partial_{i} d_{j}^{1}+2 f_{i j}^{1}+2 f_{i l}^{1}\left(\partial_{j} \beta^{l}\right)-4 f_{i j}^{3}+2 T_{l j i} \beta^{l}=0 \\
-\left(\partial_{i} d_{l}^{6}\right)\left(\partial_{j} \partial_{k} \beta^{l}\right)-d_{l}^{6}\left(\partial_{j} \partial_{k} \partial_{i} \beta^{l}\right)+6 \partial_{i} e_{j k}^{1}-\partial_{i} e_{j k}^{3}-\partial_{k} e_{i j}^{5}-f_{i l}^{1}\left(\partial_{j} \partial_{k} \beta^{l}\right)-\left(\partial_{i} \beta^{l}\right)\left(\partial_{l} f_{j k}^{2}\right) \\
-\beta^{l}\left(\partial_{l} \partial_{i} f_{j k}^{2}\right)-2\left(\partial_{i} f_{k l}^{2}\right)\left(\partial_{j} \beta^{l}\right)-2 f_{k l}^{2}\left(\partial_{j} \partial_{i} \beta^{l}\right)+2 k_{j k i}^{1}-\partial_{i}\left(k_{k l j}^{1} \beta^{l}\right)+k_{j k l}^{1}\left(\partial_{i} \beta^{l}\right) \\
-4 k_{j k i}^{2}-\beta^{l}\left(\partial_{l} T_{j k i}\right)-T_{j k l}\left(\partial_{i} \beta^{l}\right)-2 T_{l j i}\left(\partial_{k} \beta^{l}\right)+4 q_{l i j k} \beta^{l}+\partial_{i} \partial_{j} \partial_{k}\left(6 \eta^{10}-\eta^{12}\right)=0
\end{gathered}
$$

Open Access. This article is distributed under the terms of the Creative Commons Attribution License (CC-BY 4.0), which permits any use, distribution and reproduction in any medium, provided the original author(s) and source are credited.

\section{References}

[1] M.J. Duff, Twenty years of the Weyl anomaly, Class. Quant. Grav. 11 (1994) 1387 [hep-th/9308075] [INSPIRE].

[2] A.B. Zamolodchikov, Irreversibility of the Flux of the Renormalization Group in a $2 D$ Field Theory, JETP Lett. 43 (1986) 730 [INSPIRE].

[3] J.L. Cardy, Is There a c Theorem in Four-Dimensions?, Phys. Lett. B 215 (1988) 749 [INSPIRE].

[4] H. Osborn, Derivation of a Four-dimensional c Theorem, Phys. Lett. B 222 (1989) 97 [INSPIRE].

[5] I. Jack and H. Osborn, Analogs for the c Theorem for Four-dimensional Renormalizable Field Theories, Nucl. Phys. B 343 (1990) 647 [INSPIRE].

[6] H. Osborn, Weyl consistency conditions and a local renormalization group equation for general renormalizable field theories, Nucl. Phys. B 363 (1991) 486 [INSPIRE].

[7] Z. Komargodski and A. Schwimmer, On Renormalization Group Flows in Four Dimensions, JHEP 12 (2011) 099 [arXiv: 1107.3987] [INSPIRE].

[8] Z. Komargodski, The Constraints of Conformal Symmetry on RG Flows, JHEP 07 (2012) 069 [arXiv: 1112.4538] [INSPIRE].

[9] L. Bonora, P. Cotta-Ramusino and C. Reina, Conformal Anomaly and Cohomology, Phys. Lett. B 126 (1983) 305 [INSPIRE]. 
[10] L. Bonora, P. Pasti and M. Bregola, Weyl Cocycles, Class. Quant. Grav. 3 (1986) 635 [INSPIRE].

[11] J.-F. Fortin, B. Grinstein and A. Stergiou, Limit Cycles and Conformal Invariance, JHEP 01 (2013) 184 [arXiv: 1208.3674] [INSPIRE].

[12] M.A. Luty, J. Polchinski and R. Rattazzi, The a-theorem and the Asymptotics of $4 D$ Quantum Field Theory, JHEP 01 (2013) 152 [arXiv:1204.5221] [INSPIRE].

[13] I. Jack and H. Osborn, Constraints on RG Flow for Four Dimensional Quantum Field Theories, Nucl. Phys. B 883 (2014) 425 [arXiv:1312.0428] [InSPIRE].

[14] F. Baume, B. Keren-Zur, R. Rattazzi and L. Vitale, The local Callan-Symanzik equation: structure and applications, JHEP 08 (2014) 152 [arXiv:1401.5983] [INSPIRE].

[15] Y. Nakayama, Consistency of local renormalization group in D $=3$, Nucl. Phys. B $\mathbf{8 7 9}$ (2014) 37 [arXiv: 1307.8048] [INSPIRE].

[16] A. Stergiou, D. Stone and L.G. Vitale, Constraints on Perturbative RG Flows in Six Dimensions, JHEP 08 (2016) 010 [arXiv: 1604.01782] [INSPIRE].

[17] R. Auzzi and B. Keren-Zur, Superspace formulation of the local RG equation, JHEP 05 (2015) 150 [arXiv:1502.05962] [INSPIRE].

[18] J. Gomis, P.-S. Hsin, Z. Komargodski, A. Schwimmer, N. Seiberg and S. Theisen, Anomalies, Conformal Manifolds and Spheres, JHEP 03 (2016) 022 [arXiv:1509.08511] [INSPIRE].

[19] Y. Nakayama, Scale invariance vs conformal invariance, Phys. Rept. 569 (2015) 1 [arXiv: 1302.0884] [INSPIRE].

[20] G.M. Shore, The $c$ and a-theorems and the Local Renormalisation Group, arXiv:1601.06662 [INSPIRE].

[21] D. Anselmi, D.Z. Freedman, M.T. Grisaru and A.A. Johansen, Nonperturbative formulas for central functions of supersymmetric gauge theories, Nucl. Phys. B 526 (1998) 543 [hep-th/9708042] [INSPIRE].

[22] D. Anselmi, Anomalies, unitarity and quantum irreversibility, Annals Phys. 276 (1999) 361 [hep-th/9903059] [INSPIRE].

[23] D. Anselmi, Inequalities for trace anomalies, length of the $R G$ flow, distance between the fixed points and irreversibility, Class. Quant. Grav. 21 (2004) 29 [hep-th/0210124] [INSPIRE].

[24] I. Adam, I.V. Melnikov and S. Theisen, A Non-Relativistic Weyl Anomaly, JHEP 09 (2009) 130 [arXiv:0907.2156] [INSPIRE].

[25] M. Baggio, J. de Boer and K. Holsheimer, Anomalous Breaking of Anisotropic Scaling Symmetry in the Quantum Lifshitz Model, JHEP 07 (2012) 099 [arXiv:1112.6416] [INSPIRE].

[26] T. Griffin, P. Hořava and C.M. Melby-Thompson, Conformal Lifshitz Gravity from Holography, JHEP 05 (2012) 010 [arXiv:1112.5660] [INSPIRE].

[27] I. Arav, S. Chapman and Y. Oz, Lifshitz Scale Anomalies, JHEP 02 (2015) 078 [arXiv: 1410.5831] [INSPIRE].

[28] I. Arav, S. Chapman and Y. Oz, Non-Relativistic Scale Anomalies, JHEP 06 (2016) 158 [arXiv: 1601.06795] [INSPIRE]. 
[29] S. Pal and B. Grinstein, Weyl Consistency Conditions in Non-Relativistic Quantum Field Theory, arXiv: 1605.02748 [INSPIRE].

[30] S. Deser and A. Schwimmer, Geometric classification of conformal anomalies in arbitrary dimensions, Phys. Lett. B 309 (1993) 279 [hep-th/9302047] [INSPIRE].

[31] K. Jensen, Anomalies for Galilean fields, arXiv:1412.7750 [INSPIRE].

[32] R. Auzzi and G. Nardelli, Heat kernel for Newton-Cartan trace anomalies, JHEP 07 (2016) 047 [arXiv: 1605. 08684] [INSPIRE].

[33] R. Auzzi, S. Baiguera and G. Nardelli, On Newton-Cartan trace anomalies, JHEP 02 (2016) 003 [Erratum ibid. 1602 (2016) 177] [arXiv: 1511.08150] [INSPIRE].

[34] D.T. Son and M. Wingate, General coordinate invariance and conformal invariance in nonrelativistic physics: Unitary Fermi gas, Annals Phys. 321 (2006) 197 [cond-mat/0509786] [INSPIRE].

[35] C. Hoyos and D.T. Son, Hall Viscosity and Electromagnetic Response, Phys. Rev. Lett. 108 (2012) 066805 [arXiv: 1109.2651] [INSPIRE].

[36] D.T. Son, Newton-Cartan Geometry and the Quantum Hall Effect, arXiv:1306.0638 [INSPIRE].

[37] M. Geracie, D.T. Son, C. Wu and S.-F. Wu, Spacetime Symmetries of the Quantum Hall Effect, Phys. Rev. D 91 (2015) 045030 [arXiv:1407.1252] [INSPIRE].

[38] K. Jensen, On the coupling of Galilean-invariant field theories to curved spacetime, arXiv: 1408.6855 [INSPIRE].

[39] J. Hartong, E. Kiritsis and N.A. Obers, Schrödinger Invariance from Lifshitz Isometries in Holography and Field Theory, Phys. Rev. D 92 (2015) 066003 [arXiv:1409.1522] [INSPIRE].

[40] J. Hartong, E. Kiritsis and N.A. Obers, Lifshitz space-times for Schrödinger holography, Phys. Lett. B 746 (2015) 318 [arXiv:1409.1519] [INSPIRE].

[41] J. Hartong, E. Kiritsis and N.A. Obers, Field Theory on Newton-Cartan Backgrounds and Symmetries of the Lifshitz Vacuum, JHEP 08 (2015) 006 [arXiv: 1502.00228] [INSPIRE].

[42] C. Duval, G. Burdet, H.P. Kunzle and M. Perrin, Bargmann Structures and Newton-cartan Theory, Phys. Rev. D 31 (1985) 1841 [INSPIRE]. 